\title{
Posterior Semicircular Canal Dehiscence Presenting with Recurrent Positional Vertigo
}

\author{
Dusan Pavlovic* \\ Hearing and Balance Center Belgrade, Europe
}

Submission: November 12, 2016; Published: November 22, 2016

*Corresponding author: Dusan Pavlovic, Hearing and Balance Center Belgrade, Gospodar Jovanova 9, Europe, Tel: +38163227973; Email: drdusan.pavlovic@gmail.com

\begin{abstract}
Vestibular symptoms to sound should arise suspicion for semicircular canal dehiscence (SCD) syndrome, especially when Tullio phenomenon or Hennebert's sign are present. But diverse clinical presentation makes this diagnosis not straightforward. Repeating episodes of positional vertigo in a patient with posterior semicircular canal dehiscence is herein presented case. Inconsistent history of patients with SCD makes this diagnosis challenging. BPPV has been described in various inner ear disorders but not yet in the SCD syndrome.
\end{abstract}

\section{Introduction}

Patients with SCD have a diverse history. The symptoms mostly suggestive for dehiscence are vestibular symptoms to sound or pressure changes, but chronic disequilibrium and audiological symptoms of autophony, aural fullness, hyperacusis, hearing loss and tinnitus are very frequently encountered also [1-3]. Some patients can hear their own eye movements and such hyperacusis to sounds transmitted through bone are called conductive hyperacusis [4]. It's still unclear why in some SCD patients no vestibular symptoms are present, but only auditory [4]. Characteristic audiogram for the SCD is conductive hearing loss, mostly pronounced in low tones with decreased bone conduction threshold [2,4]. But other forms of audiograms has been described also [1]. Air-bone gap with normal tympanograms and acoustic reflex present should raise suspicion for the third window lesion [5]. VEMPs are very sensitive detector of the third window lesion and show decreased threshold on the side of the lesion [2]. CT scan studies showed that the dehiscence of the posterior canal is almost as frequent as the superior one [6].

The symptoms of posterior canal dehiscence seem to be almost the same as superior canal dehiscence [7]. Etiology of the superior canal dehiscence is still under debate, but it's believed that developmental failure of outer bone layers over superior canal, makes it a predilection place for dehiscence [8]. A posterior canal dehiscence mainly results from jugular bulb erosion, while a lateral canal dehiscence is usually from cholesteatoma erosion [7]. SCD represents a third window on a bony labyrinth. It makes sound pressure from the oval window to dissipate and leak through dehiscence resulting in hearing loss. At the same time third window increases pressure difference between the two sides of cochlear partition, making cochlea more sensitive to bone conduction [3]. In the normal ear only strong sound stimuli can stimulate sensory epithelium of the maculae which are the bases of VEMP testing. But in the patient with the dehiscence of the semicircular canal, acoustic flow to the place of small impedance (third window) deflects also vestibular hair cells in the affected canal and makes cupula sensitive to sound stimulus [9]. Recorded eye movements are in a plane of dehiscent canal [10].

\section{Case Report}

A 49 year old man presented with a history of positional vertigo. Short vertigo spells were mostly pronounced in the morning when getting up and were repeating with bending and other provocative positions characteristic for BPPV. Usually these episodes of positional vertigo were lasting for a week and repeated two times per year. It has started few years ago and the last one was a month ago. Also, he has tinnitus (continuous high pitched) in the right ear for 5-6 years and during that period he noticed slowly progressive diminishing of hearing in the right ear. He denied other audiological symptoms like aural fullness, autophony and pressure or sound induced vertigo.

Neurotological exam was normal and Dix-Hallpike test was negative. VHIT demonstrated good VOR gain in all six canals. Audiometry on the right ear showed mild to moderate down 
sloping curve with mixed, predominantly conductive type of hearing loss with bone conduction thresholds in low tones below normal (Figure 1). Since otoscopy and tympanometry didn't show any middle ear pathology (Figure 2), cVEMPs were performed and showed normal amplitudes and latencies at both sides (Figure 3). It was a sign to ask for a CT scan which confirmed third window lesion - the posterior canal dehiscence due to high jugular bulb (Figure 4).
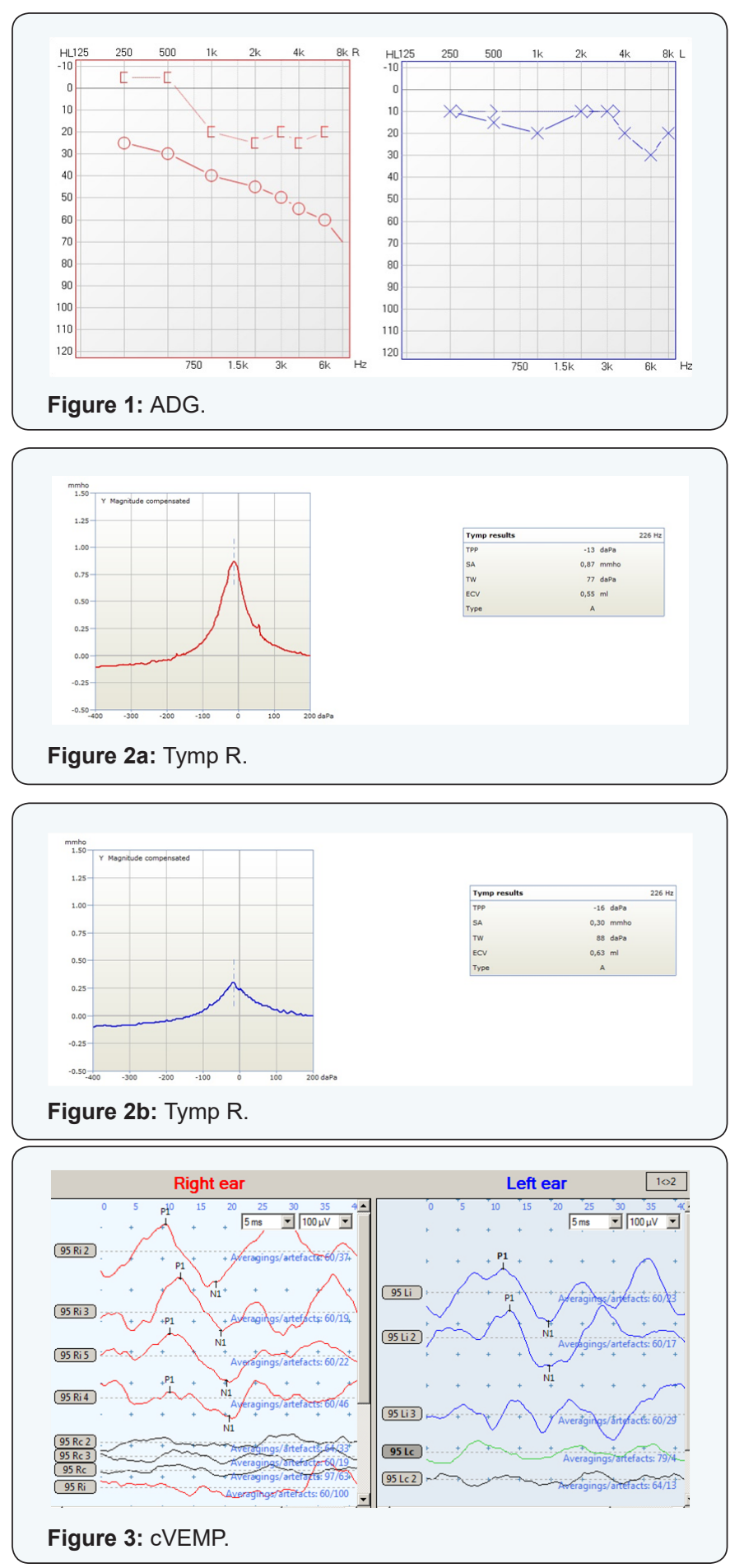

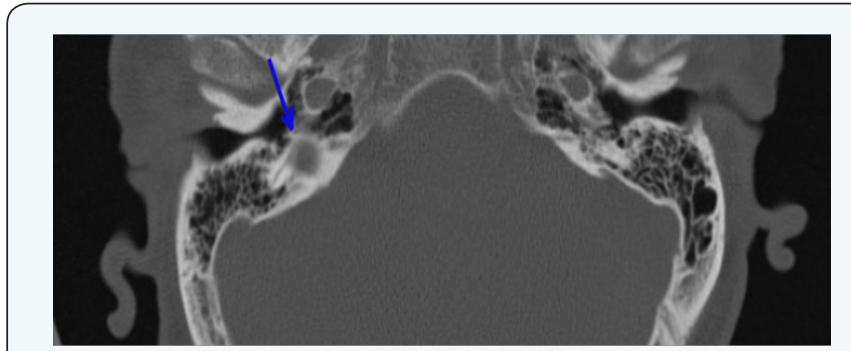

Figure 4a: СT Axial.

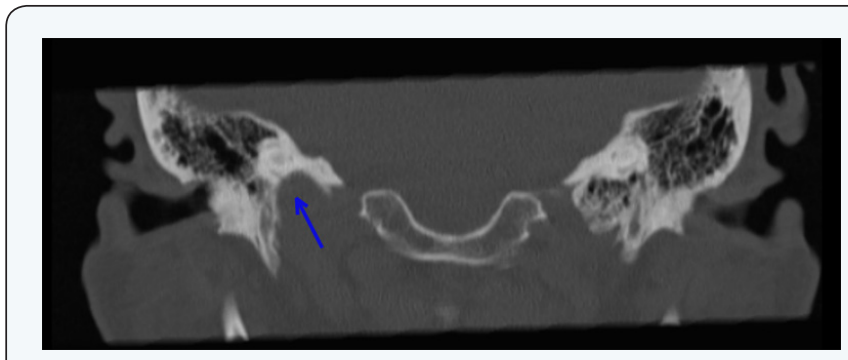

Figure 4b: CT Coronal.

\section{Discussion}

The history of the presented patient corresponds to the recurrent BPPV. Additionally his audiological symptoms of continuous high pitched tinnitus with mild to moderate hearing loss didn't arouse suspicion for a SCD. But conductive hearing loss without obvious middle ear pathology especially with decreased bone conduction thresholds in low tones was indication to check for the VEMPs. Presence of vestibulo-cervical reflex pointed that the air-bone gap is of inner ear origin. Finally, CT scan confirmed dehiscence of the posterior semicircular due to the high jugular bulb. Discovered dehiscence explains the right sided hearing loss and tinnitus, but recurrent episodes of positional vertigo haven't been described in SCD syndrome yet. As there is no proof for canalolithiasis of the posterior canal it can be only assumed according to the characteristic history that he had episodes of BPPV. The explanation might be in the pressure of the jugular

bulb threw dehiscent posterior canal and influence on fluid dynamics in it.

Another would be that acoustic flow through vestibular part makes more frequent detachment of otoconia. The second explanation is less probable in this patient as he hasn't have the vestibular symptoms to sounds

or pressure stimuli. VHIT showed normal VOR gains in all six canals including the dehiscente one. The reduced VOR gain could be expected in dehiscence bigger than $5 \mathrm{~mm}$ [10]. Dehiscence can be successfully cured surgically, but it's usually not recommended in cases with mild symptoms as is the case of the presented patient [4]. The progression of the high jugular bulb is unpredictable and it's advised to monitor this patient. 


\section{Conclusion}

The characteristic signs of SCD as Tullio phenomenon and Henneberth's sign are not seen in every patient with SCD syndrome. Also, inconsistent history of the patients with SCD, makes this diagnosis challenging and probably often undiscovered [2]. Whenever conductive hearing loss is without obvious signs of middle ear pathology it should be checked if the air-bone gap is due to inner ear pathology as is a case of a SCD. VEMPs are very sensitive for the presence of the third window lesion [2]. It has been suggested that oVEMPs are even more sensitive for SCD than cVEMPs [11,12]. Dehiscence is mostly prevalent on the superior canal but it's not so infrequent finding in the posterior one also [6]. Usually it's due to high jugular bulb $[13,14]$. The unusual cases of multi dehiscent bony labyrinth has been described also $[15,16]$. Recurrent BPPV has been seen in various inner ear disorders but hasn't been described in patients with SCD syndrome yet, except the unusual case of a patient who developed posterior canal BPPV after canal plugging [16,17].

\section{References}

1. Chi FL, Ren DD, Dai CF (2010) Variety of audiologic manifestations in patients with superior semicircular canal dehiscence. Otol Neurotol 31(1): 2-10.

2. Zhou G, Gopen Q Poe DS (2007) Clinical and diagnostic characterization of canal dehiscence syndrome: a great otologic mimicker. Otol Neurotol 28(7): 920-926.

3. Yuen HW, Eikelboom RH, Atlas MD (2009) Auditory manifestations of superior semicircular canal dehiscence. Otol Neurotol 30(3): 280-285.

4. Minor LB (2005) Clinical manifestations of superior semicircular canal dehiscence. Laryngoscope 115(10): 1717-1727.

5. Merchant SN, Rosowski JJ (2008) Conductive hearing loss caused by third-window lesions of the inner ear. Otol Neurotol 29(3): 282-289.

6. Krombach GA, DiMartino E, Schmitz-Rode T, Prescher A, Haage P, et al. (2003) Posterior semicircular canal dehiscence: a morphologic cause of vertigo similar to superior semicircular canal dehiscence. Eur Radiol 13(6): 1444-1450.
7. Gopen Q, Zhou G, Poe D, Kenna M, Jones D (2010) Posterior semicircular canal dehiscence: first reported case series. Otol Neurotol 31(2): 339344 .

8. Carey JP, Minor LB, Nager GT (2000) Dehiscence or thinning of bone overlying the superior semicircular canal in a temporal bone survey. Arch Otolaryngol Head Neck Surg 126(2): 137-147.

9. Watson SR, Halmagyi GM, Colebatch JG (2000) Vestibular hypersensitivity to sound (Tullio phenomenon): structural and functional assessment. Neurology 54(3): 722-728.

10. Cremer PD, Minor LB, Carey JP, Della Santina CC (2000) Eye movements in patients with superior canal dehiscence syndrome align with the abnormal canal. Neurology 55(12): 1833-1841.

11. Zuniga MG, Janky KL, Nguyen KD, Welgampola MS, Carey JP (2013) Ocular versus cervical VEMPs in the diagnosis of superior semicircular canal dehiscence syndrome. Otol Neurotol 34(1): 121-126.

12. Janky KL, Nguyen KD, Welgampola M, Zuniga MG, Carey JP (2013) Airconducted oVEMPs provide the best separation between intact and superior canal dehiscent labyrinths. Otol Neurotol 34(1): 127-134.

13. Park JJ, Shen A, Loberg C, Westhofen M (2015) The relationship between jugular bulb position and jugular bulb related inner ear dehiscence: a retrospective analysis. Am J Otolaryngol 36(3): 347-351.

14. Friedmann DR, Le BT, Pramanik BK, Lalwani AK (2010) Clinical spectrum of patients with erosion of the inner ear by jugular bulb abnormalities. Laryngoscope 120(2): 365-372.

15. Nischal G Kundaragi, Srinivasa Mudali, Bulabai Karpagam, Rathna Priya (2014) Intracranially protruded bilateral posterior and superior SCCs with multiple dehiscences in a patient with positional vertigo: CT and MR imaging findings and review of literature. Indian Journal of Radiology and Imaging 24(4): 406-409.

16. Dang PT, Kennedy TA, Gubbels SP (2014) Simultaneous, unilateral plugging of superior and posterior semicircular canal dehiscences to treat debilitating hyperacusis. J Laryngol Otol 128(2): 174-178.

17. Pérez P, Franco V, Cuesta P, Aldama P, Alvarez MJ, et al. (2012) Recurrence of benign paroxysmal positional vertigo. Otol Neurotol 33(3): 437-443.

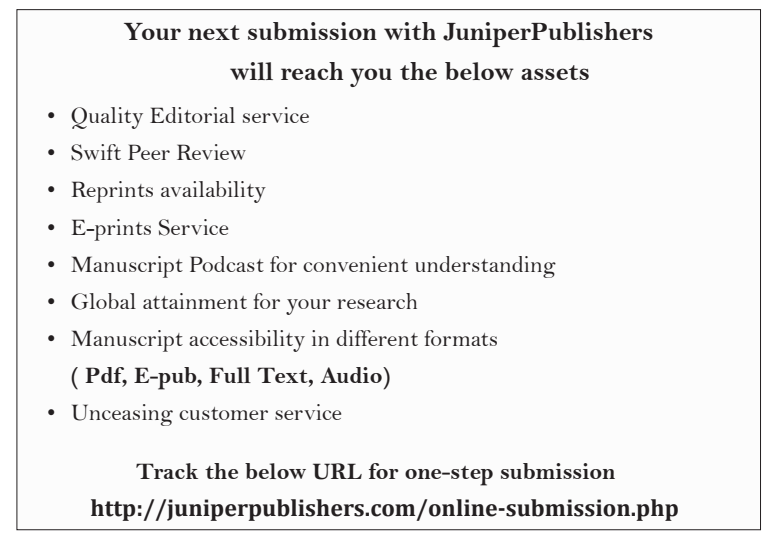

